NONSTANDARD FINITE DIFFERENCE MODELS OF DIFFERENTIAL EQUATIONS 


\section{NONSTANDARD FINITE DIFFERENCE MODELS OF DIFFERENTIAL EQUATIONS}

\section{Ronald E. Mickens}

\section{Callaway Professor of Physics}

Clark Atlanta University 
Published by

World Scientific Publishing Co. Pte. Ltd.

P O Box 128, Farrer Road, Singapore 9128

USA office: Suite 1B, 1060 Main Street, River Edge, NJ 07661

UK office: 73 Lynton Mead, Totteridge, London N20 8DH

\section{Library of Congress Cataloging-in-Publication Data}

Mickens, Ronald E., 1943-

Nonstandard finite difference models of differential equations /

Ronald E. Mickens.

p. $\quad \mathrm{cm}$.

Includes bibliographical references and index.

ISBN 9810214588

1. Finite differences. 2. Differential equations -- Numerical

solutions. I. Title.

QA431.M428 1994

515'.624--dc20

93-37665

CIP

Copyright @ 1994 by World Scientific Publishing Co. Pte. Ltd.

All rights reserved. This book, or parts thereof, may not be reproduced in any form or by any means, electronic or mechanical, including photocopying, recording or any information storage and retrieval system now known or to be invented, without written permission from the Publisher.

For photocopying of material in this volume, please pay a copying fee through the Copyright Clearance Center, Inc., 27 Congress Street, Salem, MA 01970, USA.

Printed in Singapore by JBW Printers \& Binders Pte. Ltd. 
This book is dedicated to my wife

Maria, my son

James Williamson, my daughter

Leah maria. 


\section{Preface}

This book was written in response to a large number of requests for copies of the author's papers on nonstandard finite difference schemes for the numerical integration of differential equations. The book provides a general summary of the methods used for the construction of such schemes. The major goal is to show that discrete (finite-difference) models exist for which the elementary types of numerical instabilities do not occur. The guiding philosophy behind this work is to get the qualitative details correct while not being overly concerned, at this level of the analysis, with the quantitative numerical results. (In any case, for most applications, the values of the various step-sizes are generally determined by the physical scales of the particular phenomena being studied.) The theoretical basis of our nonstandard discrete modeling methods is centered at the concepts of "exact" and "best" finite difference schemes. A set of rules is presented for constructing nonstandard finite difference schemes. The application of these rules often leads to an "essentially" unique finite difference model for a particular differential equation. It is expected that additional rules and restrictions will be discovered as research proceeds in this area.

An important feature of this book is the illustration of the various discrete modeling principles by their application to a large number of both ordinary and partial differential equations. The background requirements needed to fully understand the text are satisfied by the knowledge acquired in an introductory course on the numerical integration of differential equations.

I thank my many colleagues for their interest in my work. Again, I am particularly grateful to Ms. Annette Rohrs for typing the complete manuscript. Both she and Maria Mickens provicled valuable editorial assistance. Finally, I thank the National Aeronautics and Space Administration for providing funds that allowed me to do research on nonstandard finite difference schemes.

Ronald E. Mickens

Atlanta, Georgia

August 1993 


\section{Table of Contents}

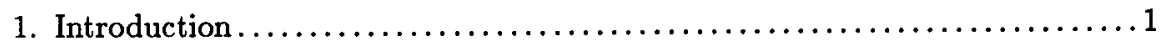

1.1 Numerical Integration......................................

1.2 Standard Finite-Difference Modeling Rules .....................

1.3 Examples ........................................... 4

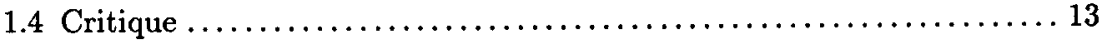

References ........................................ 14

2. Numerical Instabilities..................................... 17

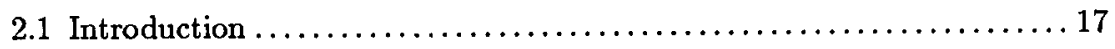

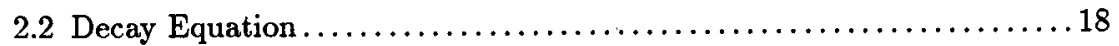

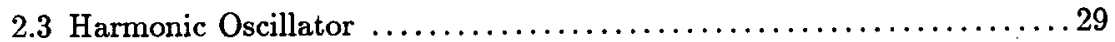

2.4 Logistic Differential Equation .............................. 35

2.5 Unidirectional Wave Equation.............................. 51

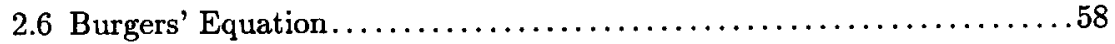

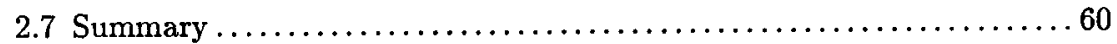

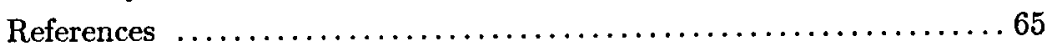

3. Nonstandard Finite-Difference Schemes $\ldots \ldots \ldots \ldots \ldots \ldots \ldots \ldots \ldots \ldots 68$

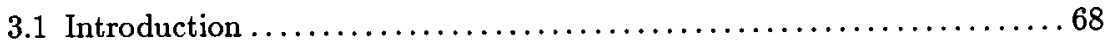

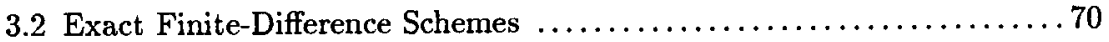

3.3 Examples of Exact Schemes............................... 72

3.4 Nonstandard Modeling Rules...............................81

3.5 Best Finite-Difference Schemes .......................... 85

References ..................................... 90

4. First-Order ODE's........................................93

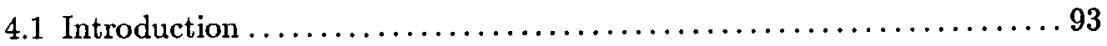

4.2 A New Finite-Difference Scheme $\ldots \ldots \ldots \ldots \ldots \ldots \ldots \ldots \ldots \ldots \ldots . . . \ldots 4$

4.3 Examples ........................................ 98

4.4 Nonstandard Schemes................................. 106

4.5 Discussion ........................................... 115

References........................................ 119 
5. Second-Order, Nonlinear Oscillator Equations $\ldots \ldots \ldots \ldots \ldots \ldots \ldots \ldots \ldots$

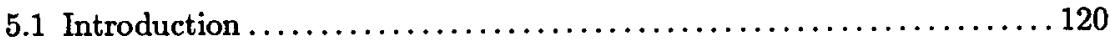

5.2 Mathematical Preliminaries ............................... 122

5.3 Conservative Oscillators................................. 124

5.4 Limit-Cycle Oscillators.................................. 132

5.5 General Oscillator Equations.............................. 137

5.6 Response of a Linear System............................ 138

References......................................... 141

6. Two First-Order, Coupled Ordinary Differential Equations ............ 144

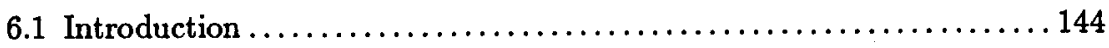

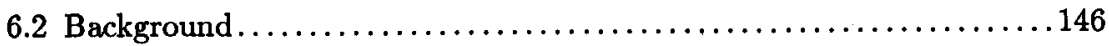

6.3 Exact Scheme for Linear Ordinary Differential Equations ........... 147

6.4 Nonlinear Equations .................................... 150

6.5 Examples............................................. 151

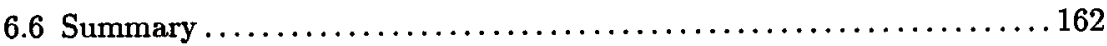

References ......................................... 163

7. Partial Differential Equations ............................... 165

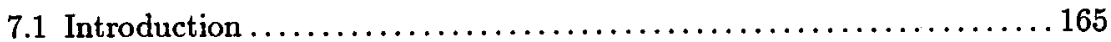

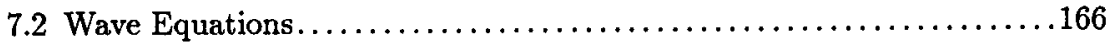

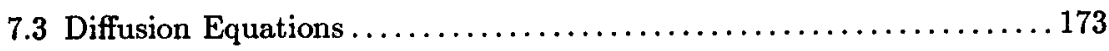

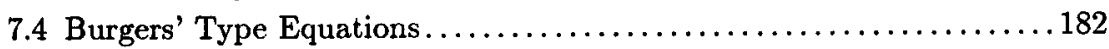

7.5 Discussion .......................................... 188

References....................................... 189

8. Schrödinger Differential Equations $\ldots \ldots \ldots \ldots \ldots \ldots \ldots \ldots \ldots \ldots \ldots \ldots \ldots \ldots$

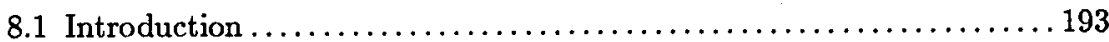

8.2 Schrödinger Ordinary Differential Equations................... 194

8.3 Schrödinger Partial Differential Equations ..................... 198

References .................................... 213

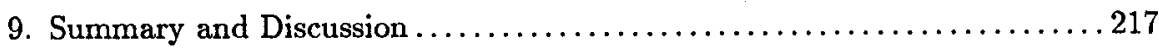

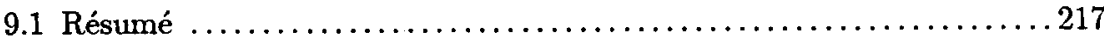

9.2 Nonstandard Modeling Rules Revisited......................219

9.3 Two Examples......................................223

9.4 Future Directions ..................................... 229

References ......................................... 230 


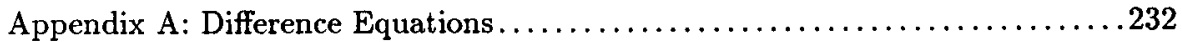

Appendix B: Linear Stability Analysis . . . . . . . . . . . . . . . . . 236

Appendix C: Discrete WKB Method ........................ 239

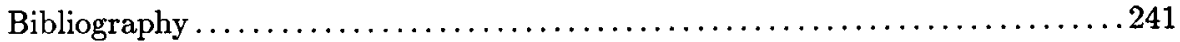

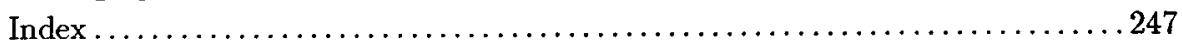

